\title{
Association between lipid accumulation product and diabetic retinopathy based on a community-based survey in Chinese with type 2 diabetes
}

This article was published in the following Dove Press journal:

Diabetes, Metabolic Syndrome and Obesity: Targets and Therapy

\author{
Jingyang $\mathrm{Wu}^{1}{ }^{1} *$ \\ Yifan Zhong ${ }^{l, *}$ \\ Song Yue' \\ Peng Guan ${ }^{2}$ \\ Guisen Zhang ${ }^{3}$ \\ Lei Liu' \\ Lei Chen'
}

'Department of Ophthalmology, The First Affiliated Hospital of China Medical University, Shenyang, I I000I, People's Republic of China; ${ }^{2}$ Department of Epidemiology, School of Public Health, China Medical University, Shenyang I I0I22, People's Republic of China; ${ }^{3}$ Department of Ophthalmology, Hohhot Chao Ju Eye Hospital, Hohhot 010000,

People's Republic of China

*These authors contributed equally to this work
Correspondence: Lei Liu

Department of Ophthalmology, The First Affiliated Hospital of China Medical

University, Shenyang II000I, People's

Republic of China

Tel +862483282630

Fax +86 2483282630

Email liuleijiao@।63.com
Purpose: Abnormal levels of lipid accumulation product (LAP) have been associated with risk of cardiovascular disease and diabetes. However, it is not clear whether LAP index is associated with diabetic retinopathy (DR). We investigated the association between LAP index and DR in Chinese adults with diabetes.

Patients and methods: We included 427 Chinese patients with type 2 diabetes aged 18 -year over who participated in a community-based cross-sectional study in Shenyang. DR was addressed on retinal photographs graded using the modified Airlie House classification. LAP was defined as (waist circumference $[\mathrm{cm}]-65) \times($ triglycerides $[\mathrm{mmol} / \mathrm{L}])$ in men, and (waist circumference $[\mathrm{cm}]-58) \times($ triglycerides $[\mathrm{mmol} / \mathrm{L}])$ in women and analyzed continuously (per SD change) and categorically (quartile 1, <30; quartile 2, 30-50; and quartile 3, >50).

Results: Prevalence of DR among the study population was $17.8 \%$. The mean level of LAP was $52.96 \pm 44.01$. Compared to quartile 1 level, both quartile 2 and 3 levels were associated with DR with multivariable odds ratio $(95 \% \mathrm{CI})$ of $0.23(0.12-0.46)$ and $0.27(0.14-0.54)$, respectively. These associations persisted when LAP was analyzed continuously $(0.57$ [0.35-0.92]).

Conclusion: Higher central lipid accumulation in Chinese diabetics is related to the lower risk of DR, suggesting that LAP may be useful for identifying type 2 diabetes mellitus patients who are at risk for DR.

Keywords: diabetic retinopathy, lipid accumulation product, diabetes

\section{Introduction}

China has become the largest country with diabetic population. Diabetic retinopathy (DR) is a common complication of diabetes and affects approximately a quarter of patients in China. ${ }^{1}$ Moreover, DR triggers preventable blindness in working-age adults in world. ${ }^{2}$ According to the increasing prevalence of diabetes, the prevention of DR is becoming a serious health problem for public. Hence, it is important to identify potential factors regarding DR.

The lipid accumulation product (LAP) level, a newly developed biomarker of central lipid accumulation, is estimated based on a combination of waist circumference (WC) and triglyceride (TG)_ levels. According to recent reports, growing evidence from both clinical-based and epidemiological studies, LAP index has been identified as an independent indicator of the risk of insulin resistance and type 2 
diabetes. $^{3,4}$ DR is a complication of diabetes; thus, it is reasonable to infer that LAP index may also have associated with DR. However, no data exist to show the relationship of LAP index with DR. To address this gap, we investigated the association between LAP index and DR in Chinese adults with diabetes who attended the Shenyang Diabetes Eye Study. ${ }^{5}$

\section{Patients and methods Study population}

Shenyang Diabetes Eye Study is a community-based study that investigates the clinical, behavioral, genetic and environmental factors associating with DR among participants with diabetes in China. The methodology of the Shenyang Diabetes Eye Study has been described previously. ${ }^{5}$ Moreover, we conducted a crosssectional study in 2014. We used the same sampling methods, questionary form, eye checking and blood testing items as Shenyang Diabetes Eye Study. Briefly, a total of 595 Chinese adults with type 2 diabetes aged $\geq 18$ years were recruited from the Fengyutan District, Shenyang, China, from August to October 2014 according to their health files in health center of Fengyutan. Our study consists of data collected from a subset of participants available LAP were included in our study (both WC and TG levels; $\mathrm{n}=434)$. Then, we excluded 7 participants for lack of historical medical treatment information (eg, use of medication), leaving 427 participants available for analysis.

Standardized interviews were done that covered demographic measures, medication use (use of insulin), lifestyle (eg, smoking and drinking) as well as behavioral factors (eg, physical excises) for diabetes management.

\section{Anthropometric tests and laboratory examinations}

Body height and weight of the participants were measured and body mass index (BMI) was calculated as weight in kilograms divided by height in meters squared. WC was measured at the umbilicus level. Blood pressure was measured using mercury sphygmomanometer according to standardized methods. ${ }^{6}$ Fasting ( $\geq 8 \mathrm{hr}$ ) venous blood samples were obtained to measure for fasting plasma glucose (FPG), hemoglobin $\mathrm{A}_{1} \mathrm{c}\left(\mathrm{HbA}_{1} \mathrm{c}\right)$, TG, and total cholesterol (TC) levels. All laboratory examinations were performed at the Endocrinology Laboratory, China Medical
University, using commercially available assays. LAP was defined as $[\mathrm{WC}(\mathrm{cm})-65] \times[\mathrm{TG}(\mathrm{mmol} / \mathrm{L})]$ in men, and $[\mathrm{WC}(\mathrm{cm})-58] \times[\mathrm{TG}(\mathrm{mmol} / \mathrm{L})]$ in women. ${ }^{7}$

\section{Definition}

Diabetes mellitus (DM) was defined as FPG $\geq 126 \mathrm{mg} / \mathrm{dL}$ (7.0 mmol/L), $\mathrm{HbA}_{1} \mathrm{c} \geq 6.5 \%$, self-reported use of antidiabetic medication or physician-diagnosed diabetes. ${ }^{8}$ Presence of type 2 diabetes was defined as physiciandiagnosed diabetes, with the information retrieved from participants' case notes.

In the current study, DR was assessed from the retinal photographs according to a standardized protocol. ${ }^{9}$ After pupillary dilation (1.0\% tropicamide), two-field (one centred on the optic disc; and the other centred on the fovea) coloured fundus photographs were taken, according to the Early Treatment for Diabetic Retinopathy Study (ETDRS) standards using a digital retinal camera (Canon CR-DGi with a 10-D SLR back; Canon, Tokyo, Japan). Fundus photographs were read in a masked manner. DR was determined to be present if any characteristic lesion was present: microaneurysms, hemorrhages, cotton wool spots, intraretinal microvascular abnormalities, hard exudates, venous bleeding, and new vessels. ${ }^{9}$

Hypertensive patients were defined as those with systolic $\mathrm{BP} \geq 140 \mathrm{mmHg}$, or diastolic $\mathrm{BP} \geq 90 \mathrm{mmHg}$, or those previously diagnosed with hypertension. Smokers were defined as those currently smoking any number of cigarettes. Drinking was defined as those who reported currently drinking five or more drinks (four or more for females) at least once. Physical exercise was defined as performing physical activity in sports or exercises at least three times per week and lasting for at least 30 mins each time in our study.

This study was approved by the Ethics Committee of the First Affiliated Hospital of China Medical University. All the participants signed an informed consent form. Our research followed the tenets of the Declaration of Helsinki.

\section{Statistical analysis}

Statistical analysis was performed by using SPSS (Version 20.0, IBM). The Chi-square test (for categorical variables) and two-independent samplet-test (for continuous variables) were oriented to compare the demographic and clinical characteristics of subjects between the two groups divided by the presence or absence of retinopathy. LAP index was analyzed as both categorical and continuous variables. LAP index was treated as a categorical variable 
which form tertiles and where moderate and high were referenced against normal levels of LAP index (quartile 1, $<30$; quartile 2, 30-50; and quartile 3, >50). Logistic regression models (Model 1: adjusted with age and gender; Model 2: adjusted with age, gender, smoking status, family history of DM, use of insulin, duration of DM, high blood pressure (HBP), BMI and $\mathrm{HbA}_{1} \mathrm{c}$ levels) were used to assess the associations of LAP index with DR. For all the tests, a $P$-value less than 0.05 was considered to be significant.

\section{Results}

There were 427 participants with type 2 diabetes involved in the current population-based study, of which 76 (17.8\%) participants had DR. Among those 76 participants with DR, $68(89.5 \%)$ had nonproliferative diabetic retinopathy (NPDR) and eight (10.5\%) had proliferative diabetic retinopathy (PDR). As shown in Table 1, participants with DR were more likely to be male, on insulin, and had longer diabetes duration, higher diastolic $\mathrm{BP}$ and $\mathrm{HbA}_{1} \mathrm{c}$ levels, lower waist and LAP levels, compared to those without DR. There were no significant differences between participants with and without DR in age, lifestyle (smoking, drinking, physical exercise and without anti-diabetes treatment), family history of diabetes, HBP, systolic BP, BMI, FPG, TG and TC levels.
In age- and gender-adjusted models (Table 2, Model 1), higher LAP index was associated with reduced risk for DR (OR: 0.53 ; 95\% CI: $0.33-0.83 ; P<0.001$ ). After adjusting for additional confounding factors (smoking status, family history of DM, use of insulin, duration of DM, HBP, BMI and $\mathrm{HbA}_{1} \mathrm{c}$ levels) in Model 2, higher LAP index was still associated with reduced risk for DR (OR: 0.57; 95\% CI: 0.35-0.92; $P<0.001)$. A consistent, significant association was found between LAP index and DR when LAP index was treated as a categorical variable in both Model 1 and Model 2 ( $P$ for trend $<0.001$ ). Moreover, when the participants with PDR were excluded, higher LAP level was associated with a lower risk of DR (OR: 0.68; 95\% CI: 0.42-0.89; $P<0.001$ ).

\section{Discussion}

To the best of our knowledge, this is the first study for investigating the association on LAP levels with presence of DR. In this community-based sample of Chinese participants with type 2 diabetes, we found that higher levels of LAP were continuously associated with a decreased presence of DR. When LAP index was analyzed categorically, the increased LAP index was still associated with decreased presence of DR.

Interestingly, our findings revealed the inverse association between LAP index and DR in Chinese adults with diabetes. The exact mechanisms underlying this inverse association are unclear. However, a similar counterintuitive association has

Table I Basic characteristic of the study participants

\begin{tabular}{|c|c|c|c|c|}
\hline Variables & Overall $(n=427)$ & DR $(n=76)$ & NDR $(n=35 I)$ & $P$-value \\
\hline Age (years) & $61.69 \pm 9.90$ & $60.13 \pm 9.59$ & $62.03 \pm 9.95$ & 0.131 \\
\hline Gender (male, \%) & 159 (37.2) & $36(47.4)$ & $123(35.0)$ & 0.045 \\
\hline Current smoking (yes, \%) & $88(20.6)$ & $14(18.4)$ & $74(21.1)$ & 0.603 \\
\hline Current drinking (yes, \%) & $38(8.9)$ & $6(7.9)$ & $32(9.1)$ & 0.735 \\
\hline Family history of DM (yes, \%) & $112(26.2)$ & $25(32.9)$ & $87(24.8)$ & 0.147 \\
\hline Duration of DM (years) & $5.72 \pm 6.12$ & $10.05 \pm 6.47$ & $4.78 \pm 5.63$ & $<0.001$ \\
\hline HBP (yes, \%) & $205(48.0)$ & $43(56.6)$ & $162(46.2)$ & 0.100 \\
\hline Use of insulin, (yes, \%) & $78(18.3)$ & $28(36.8)$ & $50(14.2)$ & $<0.001$ \\
\hline Physical exercise (yes, \%) & $31(7.3)$ & $3(3.9)$ & $28(8.0)$ & 0.230 \\
\hline Without anti-diabetes treatment (yes, \%) & $49(11.5)$ & $6(7.9)$ & $43(12.3)$ & 0.284 \\
\hline $\mathrm{SBP}(\mathrm{mmHg})$ & $136.78 \pm 13.37$ & $137.83 \pm 13.56$ & $136.55 \pm 13.34$ & 0.45 \\
\hline $\mathrm{DBP}(\mathrm{mmHg})$ & $81.5 \pm 7.91$ & $83.33 \pm 8.22$ & $81.1 \pm 7.79$ & 0.03 \\
\hline Waist $(\mathrm{cm})$ & $85.96 \pm 6.78$ & $82.61 \pm 6.55$ & $86.69 \pm 6.81$ & $<0.001$ \\
\hline BMI $\left(\mathrm{kg} / \mathrm{m}^{2}\right)$ & $24.80 \pm 2.58$ & $24.29 \pm 2.75$ & $24.90 \pm 2.54$ & 0.063 \\
\hline FPG (mmol/L) & $7.84 \pm 3.19$ & $7.97 \pm 2.82$ & $7.82 \pm 3.27$ & 0.702 \\
\hline TG (mmol/L) & $2.10 \pm 1.56$ & $1.91 \pm 1.53$ & $2.14 \pm 1.57$ & 0.247 \\
\hline $\mathrm{TC}(\mathrm{mmol} / \mathrm{L})$ & $5.02 \pm 1.02$ & $4.92 \pm 1.22$ & $5.04 \pm 0.97$ & 0.351 \\
\hline $\mathrm{HbA}, \mathrm{c}(\%)$ & $7.26 \pm 1.53$ & $7.93 \pm 1.76$ & $7.11 \pm 1.43$ & $<0.001$ \\
\hline LAP $(\mathrm{cm} \cdot \mathrm{mmol} / \mathrm{L})$ & $52.96 \pm 44.01$ & $40.76 \pm 39.60$ & $55.60 \pm 44.51$ & 0.006 \\
\hline
\end{tabular}

Abbreviations: DBP, diastolic blood pressure; SBP, systolic blood pressure; NDR, nondiabetic retinopathy; DR, diabetic retinopathy; DM, diabetes mellitus; FPG, fasting plasma glucose; HBP, high blood pressure; HbAlc, hemoglobin $\mathrm{A}_{1} \mathrm{c}$; TG, triglyceride; TC, total cholesterol; LAP, lipid accumulation product; BMI, body mass index. 
Table 2 Associations of LA index with DR

\begin{tabular}{|c|c|c|c|c|c|c|}
\hline & \multirow[t]{2}{*}{$\mathbf{n}$} & \multirow[t]{2}{*}{ DR, n (\%) } & \multicolumn{2}{|l|}{ Model I ${ }^{\mathbf{a}}$} & \multicolumn{2}{|l|}{ Model $2^{b}$} \\
\hline & & & OR $(95 \% \mathrm{CI})$ & $P$-value & OR $(95 \% \mathrm{CI})$ & P-Value \\
\hline LAP $(\mathrm{cm} \cdot \mathrm{mmol} / \mathrm{L})$ & & & & & & \\
\hline Quartile I, LAP index $<30$ & 123 & $40(32.5)$ & I & - & I & - \\
\hline Quartile 2, LAP index 30-50 & 198 & $24(12.1)$ & $0.28(0.15-0.53)$ & $<0.001$ & $0.23(0.12-0.46)$ & $<0.001$ \\
\hline Quartile 3, LAP index >50 & 106 & $12(11.3)$ & $0.28(0.15-0.5 \mathrm{I})$ & $\begin{array}{l}<0.00 \mathrm{I} \\
P \text { for trend }<0.00 \mathrm{I}\end{array}$ & $0.27(0.14-0.54)$ & $\begin{array}{l}<0.001 \\
P \text { for trend }<0.001\end{array}$ \\
\hline Per SD increase in LAP index & 427 & $76(17.8)$ & $0.53(0.33-0.83)$ & $<0.001$ & $0.57(0.35-0.92)$ & $<0.001$ \\
\hline
\end{tabular}

Notes: ${ }^{a}$ Adjusted age and gender. ${ }^{b}$ Adjusted for age, gender, smoking status, family history of DM, use of insulin, duration of DM, HBP, BMI and HbAIc levels.

Abbreviations: DR, diabetic retinopathy; BMI, body mass index; LAP, LAP, lipid accumulation product; HbAlc, hemoglobin Alc; HBP, high blood pressure; DM, diabetes mellitus.

been reported in other disease states, including chronic kidney disease, congestive cardiac failure, peripheral arterial disease, stroke risk and thromboembolism. ${ }^{10-13}$ This inverse association has been labeled as the "obesity paradox". ${ }^{14}$ On the other hand, it may not be true that higher LAP protects people by diabetes against DR, but that subjects with a lower LAP are more likely to be more severe DM (causing weight loss) and thus have an increased risk of DR. In addition, long-term duration of diabetes is associated with DR. ${ }^{15}$ Moreover, due to a lesser capacity for insulin secretion, people with long-term duration of diabetes tend to have a lower LAP as compared to those with shorter diabetes duration. This could be a key factor in explaining the inverse association of LAP index and DR.

In the current study, a higher LAP index was associated with lower risk of DR, but our finding should be interpreted with caution. It must not be made to summarize that a higher LAP index is preferable. It should be noted that many health problems including diabetes and overall increased mortality are associated with obesity. ${ }^{16}$
The LAP is an index used for evaluating lipid overaccumulation and metabolic disorder. In addition, BMI is also another biomarker of obesity, and in assessment related to DR in adults with diabetes. However, it is not clear whether BMI could affect DR. In our univariate analysis exploring the risk factors of early stage of DR, BMI index was not associated with DR. Additionally, in the multivariate regression adjusting for potential confounders (Table 3), BMI was not association with DR. This is consistent with other cohorts ${ }^{17,18}$ that showed no association between BMI and DR. However, another Singapore Malay and Indian eye study showed that higher BMI was associated with a lower incidence of DR. ${ }^{19}$ Furthermore, in another population-based cohort study, increased DR incidence was found in individuals with higher BMI. ${ }^{20}$ Hence, the actual relationship between BMI and DR warrants further studies. In order to address this gap, we investigate the association between LAP index and DR. Compared with BMI, LAP index might be better for identifying US adults at cardiovascular risks. ${ }^{7}$ Moreover, a recent cross-sectional study on 2,524 nondiabetic Chinese subjects

Table 3 Associations of LAP index with DR adjusted potential confounders with full models

\begin{tabular}{|c|c|c|c|c|}
\hline \multirow[t]{2}{*}{ Variable } & \multicolumn{2}{|l|}{ Model I } & \multicolumn{2}{|l|}{ Model $2^{b}$} \\
\hline & OR (95\%Cl) & P-value & OR (95\%CI) & $P$-value \\
\hline Age (years) & $0.984(0.959-1.010)$ & 0.223 & $0.966(0.937-0.996)$ & 0.025 \\
\hline Gender (male) & $0.660(0.397-1.099)$ & 0.66 & $0.527(0.279-0.996)$ & 0.048 \\
\hline LAP (cm mmol/L) & $0.987(0.977-0.997)$ & 0.013 & $0.987(0.976-0.998)$ & 0.02 \\
\hline Smoke (yes) & & & $0.57 \mid(0.258-\mid .268)$ & 0.169 \\
\hline Family history of DM (yes) & & & $0.927(0.497-1.729)$ & 0.811 \\
\hline Duration of DM (year) & & & 1.115 (1.060-I.I73) & $<0.001$ \\
\hline High blood pressure $(\mathrm{mmHg})$ & & & $1.745(0.963-3.161)$ & 0.066 \\
\hline $\mathrm{BMI}\left(\mathrm{kg} / \mathrm{m}^{2}\right)$ & & & $0.935(0.839-1.055)$ & 0.275 \\
\hline Use of Insulin (yes) & & & $1.288(0.635-2.613)$ & 0.483 \\
\hline $\mathrm{HbA}_{\mathrm{l}} \mathrm{c}(\%)$ & & & $1.228(1.04|-| .448)$ & 0.48 \\
\hline
\end{tabular}

Notes: ${ }^{a}$ Adjusted age and gender. ${ }^{\mathrm{b}}$ Adjusted for age, gender, smoking status, family history of DM, use of insulin, duration of DM, HBP, BMI, and HbAlc levels. Abbreviations: LAP, lipid accumulation product; DR, diabetic retinopathy; HbAlc, hemoglobin Alc; DM, diabetes mellitus; BMI, body mass index. 
showed that, compared to BMI, LAP had a greater impact on the homeostasis model assessment insulin resistance index (HOMA-IR). ${ }^{21}$ Further cohort study is still needed to identify the relevance of LAP and BMI in the causation of DR and determine which one may then be used as an effective biomarker of DR.

The strengths of the current study include a communitybased survey to minimize the selection bias and a standardized and comprehensive protocol for identifying factors. However, there were some limitations to our study. First, it was a cross-sectional study with a small sample size and the lack of healthy control subjects. Second, among patients with DR, majority had NPDR (89.5\%) stage; thus, the association between LAP index and severity of DR had not been explored extensively. Third, two-field retinal photographs were used to evaluate and monitor the presence of DR. This may have led to an underestimation of the true prevalence of DR. Finally, we were not able to adjust other potential confounders such as education and income levels as such data were not collected.

In conclusion, we showed in this population-based study that higher LAP index was associated with a lower risk of DR. As obesity and DR are complex and multifactorial traits influenced by multiple environmental and genetic factors, further studies may be warranted to investigate the multifactorial effects of LAP index on the presence of DR.

\section{Acknowledgments}

The study was supported by National Natural Science Foundation of China (81300783), Science Foundation of Liaoning Provincial Department of Education (LQNK201703) and Liaoning Xingliao Program. The funders had no role in design and conduct of the study; collection, management, analysis, and interpretation of the data; preparation, review, or approval of the manuscript; or in the decision to submit the manuscript for publication.

\section{Disclosure}

The authors report no conflicts of interest in this work.

\section{References}

1. Liu L, Wu X, Liu L, et al. Prevalence of diabetic retinopathy in mainland China: a meta-analysis. PLoS One. 2012;7(9):e45264.

2. Cheung N, Mitchell P, Wong TY. Diabetic retinopathy. Lancet. 2010;376(9735):124-136.
3. Wakabayashi I, Daimon T. A strong association between lipid accumulation product and diabetes mellitus in Japanese women and men. $J$ Atheroscler Thromb. 2014;21(3):282-288.

4. Xiang S, Hua F, Chen L, Tang Y, Jiang X, Liu Z. Lipid accumulation product is related to metabolic syndrome in women with polycystic ovary syndrome. Exp Clin Endocrinol Diabetes. 2013;121 (2):115-118.

5. Liu L, Wu J, Yue S, et al. Incidence density and risk factors of diabetic retinopathy within type 2 diabetes: a five-year cohort study in China (Report 1). Int J Environ Res Public Health. 2015;12 (7):7899-7909.

6. Chobanian AV, Bakris GL, Black HR, et al. The seventh report of the joint national committee on prevention, detection, evaluation, and treatment of high blood pressure: the JNC 7 report. JAMA. 2003;289(19):2560-2572.

7. Kahn HS. The "lipid accumulation product" performs better than the body mass index for recognizing cardiovascular risk: a population-based comparison. BMC Cardiovasc Disord. 2005;5:26.

8. American Diabetes Association. Standards of medical care in diabetes-2014. Diabetes Care. 2014;37(Suppl 1):S14-S80.

9. Wong TY, Klein R, Islam FM, et al. Diabetic retinopathy in a multi-ethnic cohort in the United States. Am $J$ Ophthalmol. 2006;141(3):446-455.

10. Beddhu S, Pappas LM, Ramkumar N, Samore M. Effects of body size and body composition on survival in hemodialysis patients. $J \mathrm{Am}$ Soc Nephrol. 2003;14(9):2366-2372.

11. Li W, Katzmarzyk PT, Horswell R, et al. Body mass index and stroke risk among patients with type 2 diabetes mellitus. Stroke. 2015;46 (1):164-169.

12. Oreopoulos A, Padwal R, Kalantar-Zadeh K, Fonarow GC, Norris CM, McAlister FA. Body mass index and mortality in heart failure: a meta-analysis. Am Heart J. 2008;156(1):13-22.

13. Romero-Corral A, Montori VM, Somers VK, et al. Association of bodyweight with total mortality and with cardiovascular events in coronary artery disease: a systematic review of cohort studies. Lancet. 2006;368(9536):666-678.

14. Carnethon MR, Rasmussen-Torvik LJ, Palaniappan L. The obesity paradox in diabetes. Curr Cardiol Rep. 2014;16(2):446.

15. Tan GS, Gan A, Sabanayagam C, et al. Ethnic differences in the prevalence and risk factors of diabetic retinopathy: the Singapore epidemiology of eye diseases study. Ophthalmology. 2018;125 (4):529-536.

16. Calle EE, Rodriguez C, Walker-Thurmond K, Thun MJ. Overweight, obesity, and mortality from cancer in a prospectively studied cohort of U.S. adults. N Engl J Med. 2003;348(17):1625-1638.

17. Bener A, Al-Laftah F, Al-Hamaq AO, Daghash M, Abdullatef WK. A study of diabetes complications in an endogamous population: an emerging public health burden. Diabetes Metab Syndr. 2014;8 (2):108-114

18. Manaviat MR, Rashidi M, Afkhami-Ardekani M. Four years incidence of diabetic retinopathy and effective factors on its progression in type II diabetes. Eur J Ophthalmol. 2008;18(4):572-577.

19. Chan JCY, Chee ML, Tan NYQ, Cheng CY, Wong TY, Sabanayagam C. Differential effect of body mass index on the incidence of diabetes and diabetic retinopathy in two Asian populations. Nutr Diabetes. 2018;8(1):16.

20. Bastawrous A, Mathenge W, Wing K, et al. The incidence of diabetes mellitus and diabetic retinopathy in a population-based cohort study of people age 50 years and over in Nakuru, Kenya. BMC Endocr Disord. 2017;17(1):19.

21. Xia $\mathrm{C}$, Li R, Zhang $\mathrm{S}$, et al. Lipid accumulation product is a powerful index for recognizing insulin resistance in non-diabetic individuals. Eur $J$ Clin Nutr. 2012;66 (9):1035-1038. 


\section{Publish your work in this journal}

Diabetes, Metabolic Syndrome and Obesity: Targets and Therapy is an international, peer-reviewed open-access journal committed to the rapid publication of the latest laboratory and clinical findings in the fields of diabetes, metabolic syndrome and obesity research. Original research, review, case reports, hypothesis formation, expert opinion and commentaries are all considered for publication. The manuscript management system is completely online and includes a very quick and fair peer-review system, which is all easy to use. Visit http://www.dovepress.com/testimonials.php to read real quotes from published authors. 